Article

\title{
Frequency-Domain Modeling of Harmonic Interactions in Voltage-Source Inverters with Closed-Loop Control
}

\author{
Malte John *,+(i) and Axel Mertens \\ Institute for Drive Systems and Power Electronics, Leibniz University of Hannover, 30169 Hannover, Germany; \\ mertens@ial.uni-hannover.de \\ * Correspondence: malte.john@volkswagen.de \\ + Current address: Volkswagen AG, 34219 Baunatal, Germany.
}

Received: 7 October 2020; Accepted: 5 November 2020; Published: 6 November 2020

check for updates

\begin{abstract}
Power electronic converters, together with their loads, sources, and controls, form a coupled system that includes many nonlinear interactions, for instance due to pulse-width modulation (PWM) and feedback control. In this paper we develop a complete, nonlinear modeling approach for voltage-source inverters in the frequency domain, taking into account the harmonic components introduced into the system from the inputs and from the nonlinear digital PWM. The most important contribution is a method for analyzing how these harmonics propagate through the nonlinear system in steady state. To enable this, an analytic model of PWM with arbitrary, multiple-frequency input is necessary. A revised model of Asymmetrical regularly-sampled double-edge PWM (AD-PWM) is proposed and its incorporation into the system model regarding sampling effects is discussed. The resulting nonlinear equation system is numerically and simultaneously solved, yielding the spectra of all relevant signals in the converter. The results are validated with time-domain simulations and with measurements, proving the effectiveness of the proposed approach.
\end{abstract}

Keywords: harmonic analysis; power converters; pulse-width modulation (PWM); frequency-domain model; voltage-source inverter (VSI); closed-loop control

\section{Introduction}

The accurate assessment of current and voltage harmonics of power electronic systems is studied to meet a variety of design goals, including determination of dc-link capacitor size and lifetime [1,2], compliance with grid codes [3], avoidance of the excitation of resonances [4], and design of active power filters $[5,6]$. Power electronic systems, for example the voltage-source inverter (VSI) with its passive components and control (Figure 1), form closely coupled relationships that result in a nonlinear closed-loop system. The analysis of the algebraic equation system in the frequency domain is a complex process but supports a deeper understanding of the behavior of the system. By direct solution of the equation system, the system harmonics can be assessed. 


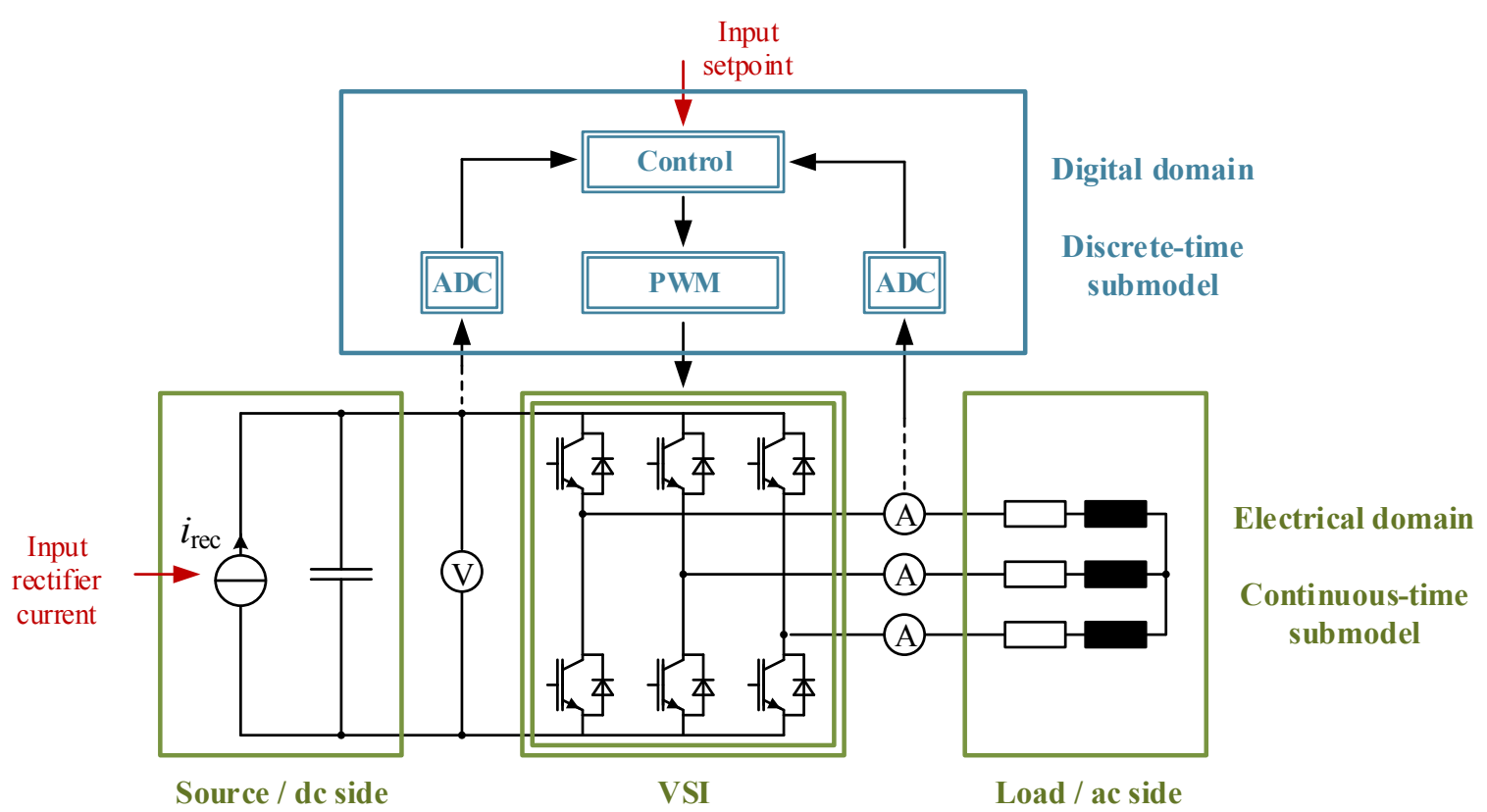

Figure 1. Topology of the example system. The two-level VSI connects a dc source to a three-phase passive load. The dc-link voltage and the ac-side currents are fed back via Analog-to-digital converters (ADCs) to the digital control, which regulates the switching state of the VSI via a pulse-width modulation (PWM). The power converter forms a closed-loop system, which is nonlinear due to the double framed blocks. The digital control in blue contains discrete-time signals and the electrical system in green is described by continuous-time signals. The system model has the rectifier current and the control setpoint as input signals displayed in red.

An important breakthrough in describing the frequency-domain behavior of switched power converters was the analysis of modulated signals. The double Fourier series expression was adapted by Bowes and Bird [7] to characterize the output spectrum of PWM converters. After further extension by Holmes [8], this method became a standard used to describe the switching spectrum of PWM VSI [9]. The double Fourier series analysis assumes in its original form:

1. A constant dc-link voltage, i.e., no interaction of dc side and ac side

2. A single frequency modulator input signal, i.e., no interaction with the control loop

3. Ideal switching of the power electronics, i.e., no dead time and lossless switches

To calculate the ac-side voltage spectrum under the influence of a variable dc-link voltage, a convolution of the switching function spectrum and the dc-link voltage spectrum can be performed. As shown by McGrath et al. [2], the dc-link current spectrum can be calculated in a similar way by using a convolution of the switching function spectrum and the ac-side current spectrum.

Subsequently, modifications to the double Fourier method as well as alternative methods evolved, characterizing the PWM output spectrum [10]. Special emphasis was given to incorporating modulator input signals that contain multiple frequency components [5,11-13] and dc-link voltage oscillations $[14,15]$. Because the interaction of the signals within the power stage of the plant and the influence of the closed-loop control system are not examined, the research question arises of how to integrate these interactions.

A common approach for modeling and analyzing PWM converters is the averaging method, introduced by Wester, Ćuk, and Middlebrook [16-18] and extended by Erickson and Maksimovic [19-21] and Hiti [22]. The averaging method is useful in developing linearized small-signal models and is commonly implemented in impedance-based models and stability analysis [23]. Corradini et al. [24] utilized this method in describing the digital control of power converters, introducing small-signal delays to consider sampling effects of the PWM and the digital control. Almér and Jönsson [25] developed a dynamic phasor model of a dc-dc converter with closed-loop control applying averaging and truncation of 
the high-frequency components. By applying small-signal assumptions, Yue et al. [26] modeled frequency components that are unsynchronized with and close to the sampling frequency and cause undesirable beat-frequency components due to aliasing.

The averaging and linearization methods truncate the high frequency components caused by the modulation and switching process. Modeling errors can result when the switching-band and the base-band components overlap and sampling effects like aliasing occur. A need for research was identified to develop a frequency-domain method that models a power electronic system including its harmonic interactions. These interactions concern the mutual dependencies between the continuous signals of the power stage, the digital signals of the control, and the influence of nonlinear effects such as modulation, sampling, and aliasing.

This article deals with the prediction of the harmonic spectrum in a converter system in the frequency domain. A special focus arises from the description of the interactions that arise in the closed control loop. We want to convey a deeper understanding of the harmonic interactions and as a result present a modeling method that enables the calculation of the spectra including their nonlinear interactions. Some modeling aspects are well known (converter model, linear components, ADC, and PI control) and they are included here for completeness of the model. The complexity and novelty of this paper lies in the PWM model's correct consideration of the sampling process and the interactions of the subsystems. This stands in contrast to the prevailing models found in the presented literature, which either model the components with their input-to-output behavior neglecting the closed-loop interactions or with a linearized behavior.

The control is developed for the example of a VSI with closed-loop current control. The influence of quantization is excluded in the models. The approach to describe the spectrum of PWM published by Song and Sarwate [11] is utilized. A review of their results for AD-PWM revealed deviations from measurement results. Therefore, we present a revised derivation of the switching spectrum for AD-PWM. The method applies for hard-switched converters using a fixed switching frequency in the linear modulation range. Furthermore, the incorporation of the PWM model into the total system model as a hybrid of discrete-time and continuous-time signals is presented with special emphasis on the consideration of sampling effects. The resulting models are evaluated with band-limited Fourier coefficients for an example case in steady state and compared with time-domain simulations and experimental results. The work presented here is a part of the author's dissertation [27].

\section{Models of Individual Components and Effects}

The example system used in this paper (Figure 1) consists of a dc source, a three-phase two-level VSI, and an RL-load. The closed-loop control of the load currents provide the ac-side voltage setpoints for the VSI, which are divided by half the measured dc-link voltage and transformed into gate signals by a pulse-width modulation (PWM) process. The influence of the dc source (e.g., a rectifier) is modeled by a current source $i_{\text {rec }}$. This enables the incorporation of the harmonic behavior of a rectifier but neglects the influence of the dc-link voltage on the rectifier.

A digital control system is assumed, because of its widespread application and to discuss the effects on the modeling approach with both discrete-time and continuous-time signals being present in the system. Analog sensors measure the required voltage and current values, which are then sampled and held by an ADC. In order to meet the control goal determined by the input setpoint signals, the control outputs duty cycles that are proportional to the required ac-side voltages. Applying a PWM to the duty cycles generates the gate signals, which control the power semiconductors. In the following sections the models of the individual components of the system are introduced.

\subsection{Switching Power Converter}

The VSI in Figure 1 consists of three half bridges. Assuming ideal switching behavior (loss-less switching and no dead time), each half bridge of phase $v \in\{1,2,3\}$ is represented by a single-pole double-throw switch with the switching function $s_{v}$, resulting in the equivalent circuit of the electrical 
subsystem in Figure 2. The switching function is used to provide the algebraic connection between the ac-side voltages $u_{\mathrm{ac} v}$ and the dc-side voltage $u_{\mathrm{dc}}$, with

$$
u_{\mathrm{ac} v}(t)=\frac{1}{2} \cdot s_{v}(t) \cdot u_{\mathrm{dc}}(t),
$$

and between the ac-side currents $i_{\mathrm{ac} v}$ and the dc-side current $i_{\mathrm{dc}}$, with

$$
i_{\mathrm{dc}}(t)=\sum_{v=1}^{3} \frac{1}{2} \cdot s_{v}(t) \cdot i_{\mathrm{ac} v}(t)
$$

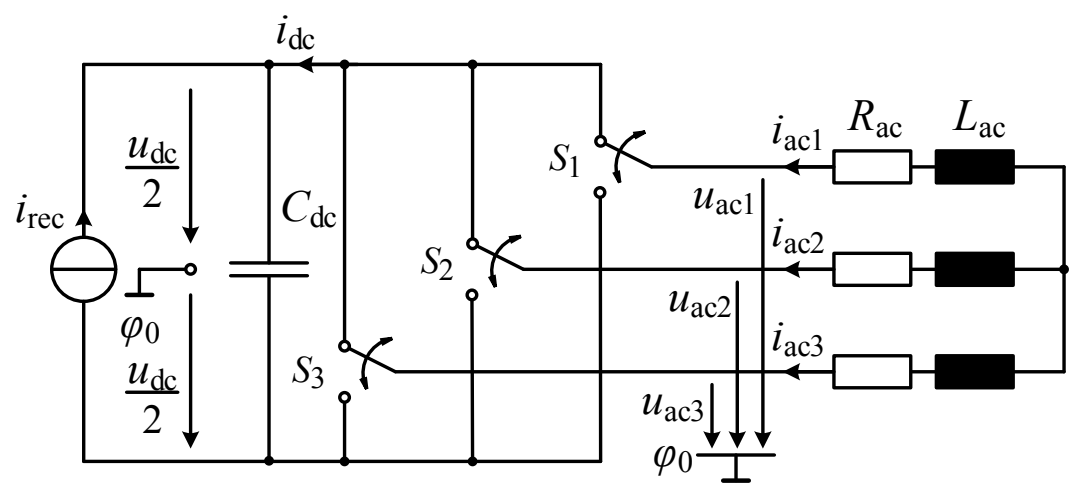

Figure 2. Equivalent circuit of the three-phase two-level VSI.

Transforming the equations to the frequency domain yields a convolution of the spectra, with

$$
\begin{aligned}
U_{\mathrm{ac} v}(f) & =\frac{1}{2} \cdot S_{v}(f) * U_{\mathrm{dc}}(f), \\
I_{\mathrm{dc}}(f) & =\sum_{v=1}^{3} \frac{1}{2} \cdot S_{v}(f) * I_{\mathrm{ac} v}(f),
\end{aligned}
$$

where $*$ denotes the convolution operator.

\subsection{Load and Source Models}

The load and source models provide the mathematical model for the connection between the voltages and currents on either side of the converter. In the common modeling case of linear passive components, the ac-side impedance representing a grid filter, a motor stray inductance or a passive load, and the dc-link capacitor are modeled as linear devices. For a symmetrical ac-side impedance, the frequency-domain expression of the ac side is

$$
\left[\begin{array}{l}
I_{\mathrm{ac} 1}(f) \\
I_{\mathrm{ac} 2}(f) \\
I_{\mathrm{ac} 3}(f)
\end{array}\right]=-\frac{1}{3} \cdot\left(R_{\mathrm{ac}}+\mathrm{j} 2 \pi f L_{\mathrm{ac}}\right) \cdot\left[\begin{array}{ccc}
2 & -1 & -1 \\
-1 & 2 & -1 \\
-1 & -1 & 2
\end{array}\right] \cdot\left[\begin{array}{l}
U_{\mathrm{ac} 1}(f) \\
U_{\mathrm{ac} 2}(f) \\
U_{\mathrm{ac} 3}(f)
\end{array}\right],
$$

where $R_{\mathrm{ac}}$ is the ac-side resistance and $L_{\mathrm{ac}}$ is the ac-side inductance.

The voltage-stiff dc link of a VSI consists of a large capacitance $C_{\mathrm{dc}}$. Its frequency-domain representation results in

$$
U_{\mathrm{dc}}(f)=\frac{1}{2 \pi f C_{\mathrm{dc}}} \cdot\left(I_{\mathrm{dc}}(f)+I_{\mathrm{rec}}(f)\right),
$$

Please note that without a further resistive component in the dc-link, (6) contains a singularity, which results in a degree of freedom for $U_{\mathrm{dc}}(0)$ in the model. 


\subsection{Analog-to-Digital Conversion and Filtering}

In order to provide feedback of the currents and voltages to the control system, the signals are sensed using analog sensors and then converted into digital signals. The block diagram of the measurement system that is used to track a continuous-time signal is depicted in Figure 3. The spectrum of the signal $X(f)$ represents $I_{\mathrm{ac} v}$ or $U_{\mathrm{dc}}$.

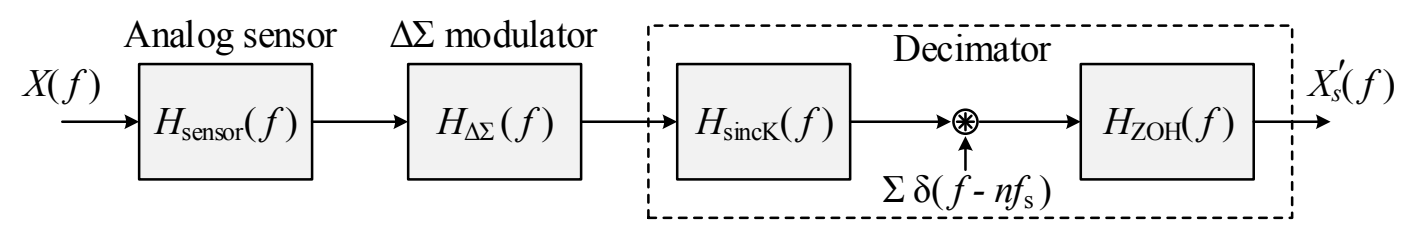

Figure 3. Structure of the measurement system.

The analog sensors are represented by a first-order low-pass filter with a cut-off frequency $f_{\text {cut }}$,

$$
H_{\text {sensor }}(f)=\frac{1}{\mathrm{j} 2 \pi f / f_{\text {cut }}+1} \text {. }
$$

In this paper, a $\Delta \Sigma$ converter is used as $A D C$, which is assumed to have a sufficiently high oversampling ratio. Therefore, its influence is low and $H_{\Delta \Sigma}(f) \approx 1$.

A decimator is used to reduce the quantization noise of the sampled signal. The decimator has the additional tasks of preventing aliasing and downsampling to the controller frequency while the signal's word size is increased. The decimation is often performed by sinc filters of order $K>1$ [28], which are described by a transfer function of

$$
H_{\text {sincK }}(f)=\left(\frac{f_{\mathrm{s}}}{f_{\Delta \Sigma}} \cdot \frac{1-\mathrm{e}^{-j 2 \pi f / f_{\mathrm{s}}}}{1-\mathrm{e}^{-j 2 \pi f / f_{\Delta \Sigma}}}\right)^{K},
$$

where $f_{\Delta \Sigma}$ is the sampling frequency of the $\Delta \Sigma$ modulator.

The output of the digital filter is sampled with the sampling frequency of the control $f_{\mathrm{s}}$. The sampling process is represented by a multiplication of the continuous signal $x(t)$ with a sequence of Dirac pulses $\delta$, which results in a continuous-time representation of a sampled signal $x_{\mathrm{s}}(t)$, with

$$
x_{\mathrm{s}}(t)=x(t) \cdot \sum_{n=-\infty}^{\infty} \delta\left(t-n / f_{\mathrm{s}}\right)
$$

Transformation of (9) into the Fourier domain results in

$$
X_{\mathrm{s}}(f)=f_{\mathrm{s}} \cdot X(f) * \sum_{n=-\infty}^{\infty} \delta\left(f-n f_{\mathrm{s}}\right)
$$

where $X_{\mathrm{s}}(f)$ is known as the Discrete-time Fourier transform (DTFT), which differs from the Continuous-time Fourier transform (CTFT) $X(f)$. This model of the sampler enables the consideration of sampling effects such as aliasing. Moreover, the sampling process is commonly synchronized with the PWM period, minimizing the influence of switching harmonics in the sensed signals [29]. This effect is included in this modeling approach and a time-shift between the PWM signal and the sampling points can easily be considered in (9) and (10).

Finally, the sampled signals are held in the control system for one control period, which is modeled with a zero-order hold $(\mathrm{ZOH})$ block, with

$$
H_{\mathrm{ZOH}}(f)=\mathrm{e}^{-\mathrm{j} \pi f T_{\mathrm{s}}} \cdot \operatorname{si}\left(\pi f T_{\mathrm{s}}\right) .
$$


The spectrum of the resulting sampled and low-pass filtered signal is written with an apostrophe $X_{\mathrm{s}}^{\prime}(f)$ to distinguish it from the original spectrum $X(f)$.

\subsection{Control System}

In this paper PI current control in the rotating reference frame is used as an example control system. The control system is shown in Figure 4 . The inputs are the sampled ac-side currents $i_{\mathrm{s}, \mathrm{ac}}^{\prime}$ and dc-link voltage $u_{\mathrm{s}, \mathrm{dc}}^{\prime}$ and the current setpoints $i_{\mathrm{s}, \mathrm{sp}}$. The outputs of the current control are the setpoint voltages in 123 coordinates $u_{\mathrm{s}, \mathrm{sp}}$, which function as inputs of the duty-cycle calculation. The outputs of the complete control block are the duty-cycle commands for the PWM process $d_{\mathrm{s}}$.

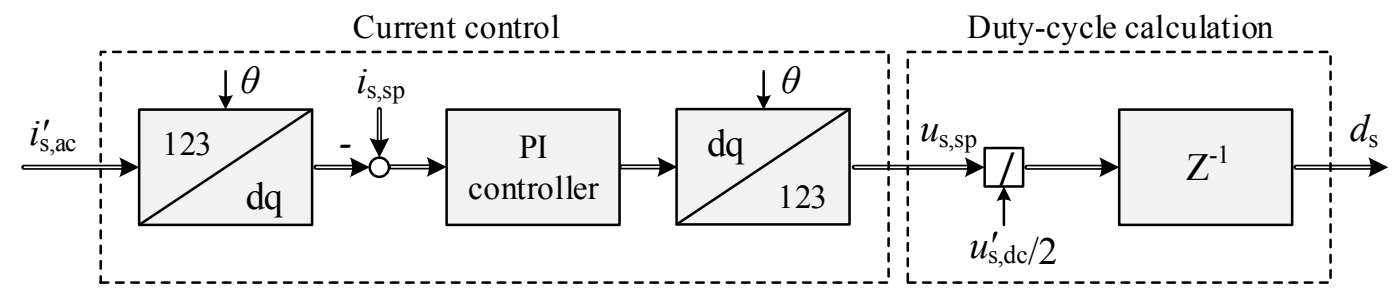

Figure 4. Block diagram for closed-loop control using PI current control and dc-link voltage feedback.

\subsubsection{Current Control}

The current control is implemented in the rotating reference frame by applying the Park transformation to the input signals. The transformation consists of the Clarke transformation and a rotation matrix. The measured ac-side current spectra are first transformed into current spectra in $\alpha \beta$ coordinates, with

$$
\left[\begin{array}{c}
I_{\mathrm{s}, \alpha}(f) \\
I_{\mathrm{s}, \beta}(f)
\end{array}\right]=\frac{2}{3} \cdot\left[\begin{array}{ccc}
1 & -\frac{1}{2} & -\frac{1}{2} \\
0 & \frac{\sqrt{3}}{2} & -\frac{\sqrt{3}}{2}
\end{array}\right] \cdot\left[\begin{array}{l}
I_{\mathrm{s}, \mathrm{ac} 1}^{\prime}(f) \\
I_{\mathrm{s}, \mathrm{ac} 2}(f) \\
I_{\mathrm{s}, \mathrm{ac} 3}^{\prime}(f)
\end{array}\right]
$$

Then a rotation into the dq reference frame is performed, which leads to a frequency shift in the current spectra [30]. For this it is assumed that the rotating angle $\theta(t)$ has a constant gradient with $\theta(t)=2 \pi f_{0} t$, where $f_{0}$ is the fundamental frequency. The current spectra in dq coordinates result in

$$
\left[\begin{array}{c}
I_{\mathrm{s}, \mathrm{d}}(f) \\
I_{\mathrm{s}, \mathrm{q}}(f)
\end{array}\right]=\frac{1}{2} \cdot\left[\begin{array}{cc}
1 & -\mathrm{j} \\
\mathrm{j} & 1
\end{array}\right] \cdot\left[\begin{array}{c}
I_{\mathrm{s}, \alpha}\left(f-f_{0}\right) \\
I_{\mathrm{s}, \beta}\left(f-f_{0}\right)
\end{array}\right]+\frac{1}{2} \cdot\left[\begin{array}{cc}
1 & \mathbf{j} \\
-\mathrm{j} & 1
\end{array}\right] \cdot\left[\begin{array}{l}
I_{\mathrm{s}, \alpha}\left(f+f_{0}\right) \\
I_{\mathrm{s}, \beta}\left(f+f_{0}\right)
\end{array}\right] .
$$

The current controller determines the setpoint voltages of the VSI in dq coordinates under the influence of the setpoint signals $I_{\mathrm{s}, \mathrm{sp}, \mathrm{d}}, I_{\mathrm{s}, \mathrm{sp}, \mathrm{q}}$, with

$$
\left[\begin{array}{l}
U_{\mathrm{s}, \mathrm{sp}, \mathrm{d}} \\
U_{\mathrm{s}, \mathrm{s}, \mathrm{q}}
\end{array}\right]=H_{\mathrm{PI}}(f) \cdot\left[\begin{array}{l}
I_{\mathrm{s}, \mathrm{sp}, \mathrm{d}}(f) \\
I_{\mathrm{s}, \mathrm{sp}, \mathrm{q}}(f)
\end{array}\right]-\left[\begin{array}{cc}
H_{\mathrm{PI}}(f) & -2 \pi f_{0} L_{\mathrm{ac}} \\
2 \pi f_{0} L_{\mathrm{ac}} & H_{\mathrm{PI}}(f)
\end{array}\right] \cdot\left[\begin{array}{c}
I_{\mathrm{s}, \mathrm{d}}(f) \\
I_{\mathrm{s}, \mathrm{q}}(f)
\end{array}\right] .
$$

A disturbance compensation of the cross-coupling of the dq axes is taken into account on the counterdiagonal of the matrix. The linear PI controllers are described by a transfer function

$$
H_{\mathrm{PI}}(f)=K_{\mathrm{P}}+\frac{K_{\mathrm{P}}}{T_{\mathrm{I}}} \cdot H_{\mathrm{I}}(f),
$$

where $K_{\mathrm{P}}$ is the proportional gain and $T_{\mathrm{I}}$ is the integrator time constant. In the case of backward Euler, the transfer function of the integrator $H_{\mathrm{I}}(f)$ is described by

$$
H_{\mathrm{I}}(f)=T_{\mathrm{S}} \cdot \frac{\mathrm{e}^{\mathrm{j} 2 \pi f T_{\mathrm{s}}}}{\mathrm{e}^{\mathrm{j} 2 \pi f T_{\mathrm{s}}}-1} .
$$


The dc component of the controller's transfer function has infinite gain, with $H_{\mathrm{I}}(f \rightarrow 0) \rightarrow$ $\infty$ and the dc components of the sampled dq currents equals the setpoint values. Consequently, the dc components of the setpoint voltages $U_{\mathrm{s}, \mathrm{sp}, \mathrm{d}}(0)$ and $U_{\mathrm{s}, \mathrm{sp}, \mathrm{q}}(0)$ cannot be calculated by (14). Their solutions result instead from the complete equation system that incorporates the interaction of the control with the physical model of the VSI and its load.

Finally, the setpoint voltages are transformed back into 123 coordinates, with

$$
\begin{gathered}
{\left[\begin{array}{l}
U_{\mathrm{s}, \mathrm{sp}, \alpha}(f) \\
U_{\mathrm{s}, \mathrm{sp}, \beta}(f)
\end{array}\right]=\frac{1}{2} \cdot\left[\begin{array}{cc}
1 & \mathrm{j} \\
-\mathrm{j} & 1
\end{array}\right] \cdot\left[\begin{array}{l}
U_{\mathrm{s}, \mathrm{sp}, \mathrm{d}}\left(f-f_{0}\right) \\
U_{\mathrm{s}, \mathrm{sp}, \mathrm{q}}\left(f-f_{0}\right)
\end{array}\right]+\frac{1}{2} \cdot\left[\begin{array}{cc}
1 & -\mathrm{j} \\
\mathrm{j} & 1
\end{array}\right] \cdot\left[\begin{array}{l}
U_{\mathrm{s}, \mathrm{sp}, \mathrm{d}}\left(f+f_{0}\right) \\
U_{\mathrm{s}, \mathrm{sp}, \mathrm{q}}\left(f+f_{0}\right)
\end{array}\right],} \\
{\left[\begin{array}{l}
U_{\mathrm{s}, \mathrm{sp}, 1}(f) \\
U_{\mathrm{s}, \mathrm{sp}, 2}(f) \\
U_{\mathrm{s}, \mathrm{sp}, 3}(f)
\end{array}\right]=\frac{2}{3} \cdot\left[\begin{array}{cc}
1 & 0 \\
-\frac{1}{2} & \frac{\sqrt{3}}{2} \\
-\frac{1}{2} & -\frac{\sqrt{3}}{2}
\end{array}\right] \cdot\left[\begin{array}{l}
U_{\mathrm{s}, \mathrm{sp}, \alpha}(f) \\
U_{\mathrm{s}, \mathrm{sp}, \beta}(f)
\end{array}\right] .}
\end{gathered}
$$

\subsubsection{Duty-Cycle Calculation}

The duty cycle is calculated by dividing the setpoint voltage of phase $v$ by half the measured dc-link voltage. The duty cycle that was calculated in sampling step $k$ is committed to the modulator within the control cycle and is applied to the switching pattern in the next control step $k+1$, with

$$
d_{v}[k+1]=\frac{u_{\mathrm{s}, \mathrm{sp}, v}[k]}{u_{\mathrm{s}, \mathrm{dc}}^{\prime}[k] / 2}
$$

The inverse convolution in the frequency domain is used to achieve the equivalent operation of the division in the time domain. Because the dc-link voltage is the state variable of an energy storage it is required that $u_{\mathrm{dc}}(t)>0, \forall t$ and $1 / u_{\mathrm{dc}}(t)$ is a slowly growing signal. This means that the requirements for the existence of the inverse convolution are fulfilled.

Lastly, the computational delay is taken into account by the delay block $\left(z^{-1}\right)$ in Figure 4. The calculation of the duty cycle thus results in

$$
D_{\mathrm{s}, v}(f)=2 \cdot H_{\text {delay }}(f) \cdot U_{\mathrm{s}, \mathrm{dc}}^{\prime}(f) * U_{\mathrm{s}, \mathrm{sp}, v}(f),
$$

where the delay is considered with

$$
H_{\text {delay }}(f)=\mathrm{e}^{-\mathrm{j} 2 \pi f T_{\mathrm{s}}} .
$$

\subsection{Model for PWM with Harmonic Input}

The switching function is determined in the PWM process. In order to include the PWM process in a frequency-domain model that considers the interaction between the control and the electrical system, an expression of the switching function spectrum

$$
S(f)=g(D(f)),
$$

as an explicit nonlinear function $g$ of the duty-cycle spectrum is needed. This can be found in the model by Song and Sarwate [11], which originates from a time-domain representation of the pulse 
pattern using the Heaviside function and is further developed into a frequency-domain representation. A model for naturally-sampled double-edge PWM (ND-PWM) is derived by Song and Sarwate with

$$
\begin{aligned}
& S_{\mathrm{ND}}(f, D)=D(f)+S_{\mathrm{c}}(f) \cdot \mathrm{e}^{-\mathrm{j} \pi f T_{\mathrm{sw}} / 2}+\sum_{m=1}^{\infty}(-1)^{m} \cdot \sum_{n=1}^{\infty} \ldots \\
& \left(\frac{(\mathrm{j} 2 m \pi)^{2 n-2}}{2^{2 n-2} \cdot(2 n-1) !} \cdot\left(D^{*(2 n-1)}\left(f+2 m f_{\mathrm{sw}}\right)+D^{*(2 n-1)}\left(f-2 m f_{\mathrm{sw}}\right)\right) \ldots\right. \\
& \left.-\frac{(\mathrm{j}(2 m-1) \pi)^{2 n-1}}{\mathrm{j} 2^{2 n-1} \cdot(2 n) !} \cdot\left(D^{*(2 n)}\left(f+(2 m-1) f_{\mathrm{sw}}\right)+D^{*(2 n)}\left(f-(2 m-1) f_{\mathrm{sw}}\right)\right)\right)
\end{aligned}
$$

where

$$
S_{\mathrm{c}}(f)=\sum_{p=0}^{\infty} \frac{2}{\mathrm{j} \pi(2 p+1)} \cdot\left[\delta\left(f-(2 p+1) f_{\mathrm{sw}}\right)-\delta\left(f+(2 p+1) f_{\mathrm{sw}}\right)\right]
$$

and $D^{* n}(f)$ denotes the Fourier transform of the duty cycle signal raised to the power of $n$ :

$$
(d(t))^{n} \circ \longrightarrow D^{* n}(f) .
$$

AD-PWM is characterized by a low total harmonic distortion level, compared to other discrete type PWM methods [9]. Due to its discrete-time property, it is easier to implement this modulation method on digital control systems in contrast to natural sampling [11]. A review of results for AD-PWM presented by Song and Sarwate ([11], (56)) revealed differences in comparison to simulation results from a validated time-domain model. A revised derivation of the analytical model for AD-PWM results in the switching function spectrum of

$$
S_{\mathrm{AD}}(f, D)=\mathrm{e}^{-\mathrm{j} \pi f T_{\mathrm{sw}} / 2} \cdot\left[S_{\mathrm{c}}(f)+\sum_{m=-\infty}^{\infty} \sum_{n=1}^{\infty} \frac{\left(\mathrm{j} \pi f T_{\mathrm{sw}}\right)^{n-1}}{2^{n} \cdot n !} \cdot D^{* n}\left(f-m f_{\mathrm{sw}}\right) \cdot\left(1-(-1)^{m+n}\right)\right] .
$$

For AD-PWM, the duty cycle of the PWM is adjusted at two sampling points per PWM cycle. Hence, the duty cycles of the two halves of a pulse differ from each other and the pulse appears asymmetrically spaced from the center of the PWM period. In comparison to Song and Sarwate ([11], (56)), the revised equation includes the representation of the two sampling points. The derivation of (26) is presented in the Appendix A. A numerical evaluation of (26) follows in Section 4.2 with a comparison with a time-domain simulation and measurement results.

The internal structure of regularly-sampled PWM can be divided into a sampler, a $\mathrm{ZOH}$, and a representation of naturally-sampled PWM. From this, two compositions of the frequency-domain model describing regularly-sampled PWM result, as demonstrated in Figure 5. Subfigure (a) represents the model described by (26), in which the input is the Fourier transform of the continuous-time duty cycle $D(f)$. The description of the sampler and the $\mathrm{ZOH}$ can be separated from the PWM model without loss of generality, using the Fourier transform of the discrete-time duty cycle $D_{\mathbf{s}}(f)$ as the input of a model of naturally-sampled double-edge PWM. This method is utilized to calculate the switching function spectrum depicted in subfigure (b). Therefore, regularly-sampled PWM is equivalent to naturally-sampled PWM when applying a "distorted" duty cycle spectrum $D_{\mathrm{s}}(f)$.

When considering feedback control, the digital controller outputs a discrete-time duty-cycle with a spectrum $D_{\mathbf{s}}(f)$ (Figure 4), which interacts with the signals of the PWM and the plant. As stated earlier, the regularly-sampled PWM model (Figure 5a) requires an input spectrum $D(f)$ of the continuous-time duty-cycle $d(t)$. However, as demonstrated in Figure 6a, a naturally-sampled PWM model may be applied to express regularly-sampled PWM under the condition that the input is the spectrum of the discrete-time signal $D_{\mathrm{s}}(f)$. Because the DTFT of the input is not band-limited, the consideration of a large spectrum is required to achieve high quality results using this model. 
a)

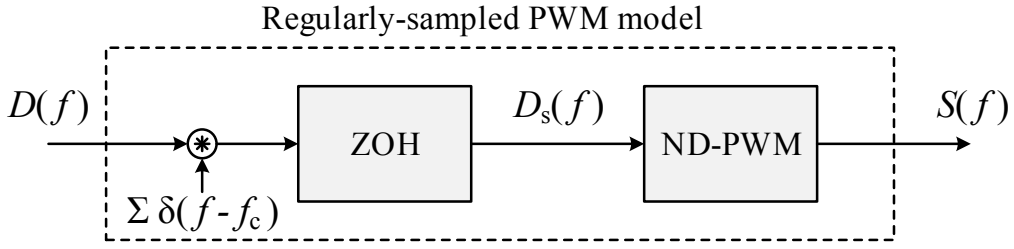

b)

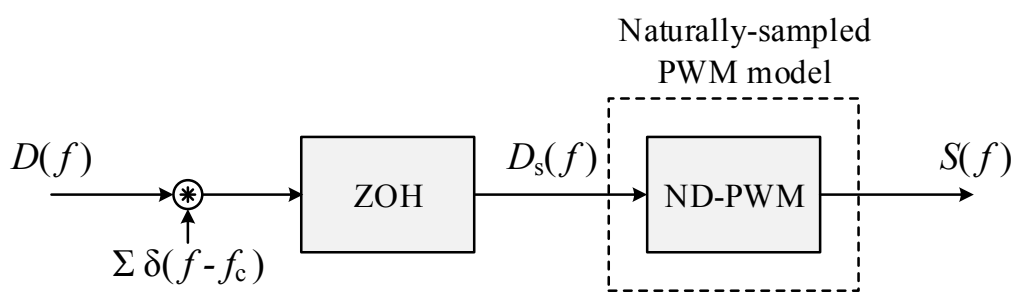

Figure 5. Equivalent methods representing regularly-sampled PWM when the input is the CTFT of the duty cycle $D(f)$ : (a) The series connection of sampler, $\mathrm{ZOH}$, and naturally-sampled PWM is combined in one mathematical expression. (b) The CTFT of the duty cycle $D(f)$ is first transferred into the DTFT of the duty cycle $D_{\mathrm{s}}(f)$ using a model of the sampler and the $\mathrm{ZOH}$. The resulting spectrum is used as the input for the naturally-sampled PWM model [27].

a)

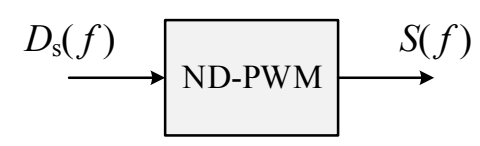

b)

b) Regularly-sampled PWM model

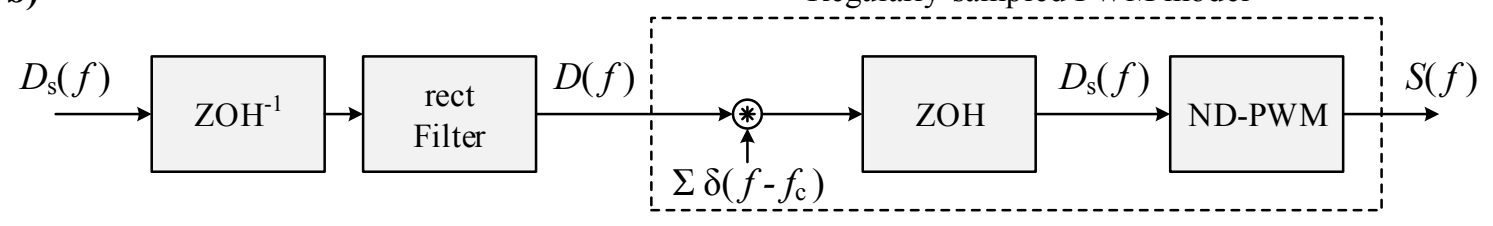

Figure 6. Equivalent methods representing regularly-sampled PWM when the input is the DTFT of the duty cycle $D_{\mathrm{s}}(f)$ : (a) A block expressing naturally-sampled PWM. (b) An inverse ZOH and a rectangular filter the ZOH block and sampler of a regularly-sampled PWM model [27].

An alternative method is presented in Figure 6b: The DTFT of the duty-cycle is converted into a CTFT, which is subsequently applied to the AD-PWM model. The benefit of this method is that input of the PWM model (dashed box in Figure 6b) is a band-limited signal and thus the consideration of a smaller spectrum is required to achieve the same quality as the method in Figure 6a. For further clarification, a numerical comparison is provided in this section. The conversion is performed by application of the inverse transfer function of the $\mathrm{ZOH}$

$$
\begin{aligned}
& H_{\mathrm{ZOH}}^{-1}(f)=\frac{\mathrm{j} 2 \pi f T_{\mathrm{c}}}{1-\mathrm{e}^{-\mathrm{j} 2 \pi f T_{\mathrm{c}}}}, \quad f \neq 0 \\
& H_{\mathrm{ZOH}}^{-1}(0)=1
\end{aligned}
$$

and the inversion of the sampling process. Since $D_{\mathbf{S}}(f)$ is the DTFT of a band-limited signal, the sampling process is inverted by a rectangular filter with

$$
H_{\text {rect }}(f)=\left\{\begin{array}{lc}
1 & \text { for }
\end{array} \quad-f_{\mathrm{c}} / 2 \leq f \leq f_{\mathrm{c}} / 2 .\right.
$$

A numerical comparison of the models in Figure 6a,b follows in Section 4.3. 


\section{System Model for Interactions}

All equations needed to describe the overall converter system form an algebraic nonlinear equation system

$$
\vec{g}(\vec{X}(f), \vec{U}(f))=\overrightarrow{0}
$$

where $\vec{X}(f)$ are the spectra of the system's signals and $\vec{U}(f)$ are the spectra of the inputs.

As a simplification, the total equation system was numerically evaluated for the steady state. This enables the representation of the periodic time-domain signals $x(t)$ by a complex Fourier series. Furthermore, the Fourier series was band-limited to a maximum order of $k_{\max }$ so that a signal $x(t)$ was approximated by

$$
x_{1}(t) \approx \sum_{k=-k_{\max }}^{k_{\max }} X_{1, k} \cdot \mathrm{e}^{j k 2 \pi f_{0} t}, \quad k \in \mathbb{Z},
$$

where $X_{1, k} \in \mathbb{C}$ is the complex Fourier coefficient of order $k$ that represents the $k$-th harmonic of the fundamental frequency $f_{0}$.

The coefficients of the band-limited Fourier series form a discrete and finite spectrum that can be collected in a vector representation

$$
\vec{X}_{1}=\left[X_{1,-k_{\max }}, . ., X_{1,-1}, X_{1,0}, X_{1,1}, . ., X_{1, k_{\max }}\right]^{\mathrm{T}} .
$$

Therefore, all input signals and system signals are represented by a vector of the length $l=2 k_{\max }+1$.

The calculation of all spectra requires the simultaneous solution of the nonlinear equation system. The equation system chosen for the following numerical evaluation describes $N=13$ signals, collected in a vector

$$
\vec{X}=\left[\vec{I}_{\mathrm{ac} 1}, \vec{I}_{\mathrm{ac} 2}, \vec{I}_{\mathrm{ac} 3}, \vec{U}_{\mathrm{dc}}, \vec{D}_{\mathrm{s}, 1}, \vec{D}_{\mathrm{s}, 2}, \vec{D}_{\mathrm{s}, 3}, \vec{I}_{\mathrm{s}, \mathrm{d}}, \vec{I}_{\mathrm{s}, \mathrm{q}}, \vec{U}_{\mathrm{s}, \mathrm{sp}, \alpha}, \vec{U}_{\mathrm{s}, \mathrm{sp}, \beta}, \vec{U}_{\mathrm{s}, \mathrm{sp}, \mathrm{d}}, \vec{U}_{\mathrm{s}, \mathrm{sp}, \mathrm{q}}\right]^{\mathrm{T}}
$$

The size of the vector is $N \cdot l$, which are given by the input signals. All other signals are described as dependent variables and are not written explicitly in the equation system. However, it is possible to calculate them directly using the signals present in $\vec{X}$. The input signals of the system are the dc-side disturbance current, the dc component of the dc-link voltage, and the current setpoint in dq coordinates. Thus the input vector $\vec{U}$ contains the spectra of the ac-current setpoints $\vec{I}_{\mathrm{s}, \mathrm{sp}, \mathrm{d}}, \vec{I}_{\mathrm{s}, \mathrm{sp}, \mathrm{q}}$, the rectifier current $\vec{I}_{\mathrm{rec}}$ and the dc component of the dc-link voltage $\vec{U}_{\mathrm{dc}}(0)$.

Further approximations are necessary to enable a numerical evaluation:

- The infinite summation in (26) is limited to $-m_{\max } \leq m \leq m_{\max }$ and $-n_{\max } \leq n \leq n_{\max }$.

- The convolution of two vectors can be represented by a multiplication of the convolution matrix of one of the vectors with the second vector. The inverse convolution in (20) is calculated by a multiplication of $\vec{U}_{\mathrm{s}, \mathrm{sp}, v}$ with the inverse of the convolution matrix $C($.$) of \vec{U}_{\mathrm{dc}}^{\prime}$. The complete convolution matrix has the size $(2 l-1) \times l$. To enable the inversion of the matrix, a truncated form of the convolution matrix is used that is square with $l \times l$. This form corresponds to the Matlab function conv when applying the option same. The truncated convolution matrix of a vector $\vec{X}$ is defined as 


$$
C(\vec{X})=\left[\begin{array}{ccccccc}
X_{0} & \ldots & X_{-k_{\max }} & & & & \\
X_{1} & \ldots & X_{\left(1-k_{\max }\right)} & X_{-k_{\max }} & & & \\
\vdots & & \vdots & \vdots & & & \\
X_{k_{\max }} & & \ldots & & & X_{-k_{\max }} \\
& & & \vdots & \vdots & & \vdots \\
& & & X_{k_{\max }} & X_{\left(k_{\max }-1\right)} & \ldots & X_{-1} \\
& & & & X_{k_{\max }} & \ldots & X_{0}
\end{array}\right]
$$

The numerical solution was executed in Matlab by applying the trust-region algorithm of the Matlab function fsolve. This algorithm was selected since it allows for the incorporation of the Jacobian matrix and a sparsity pattern. Unlike the complicated analytical derivation of the Jacobian, the analysis of a dependency between two variables is straightforward. This enables the formulation of a sparsity matrix, which indicates all partial derivatives of the input variables unequal to zero. Knowledge of the sparsity matrix enables the solver to accelerate the solution process by calculating only nonzero derivatives. In this particular case, less than $15 \%$ of the matrix elements were nonzero. By applying the sparsity pattern, the number of function calls for the numerical calculation of the Jacobian was halved.

A vector of initial values $\vec{X}_{0}$ is required for the iterative solution process, which is provided by a simple fundamental frequency model. It calculates the fundamental frequency components for ac quantities and the dc component of dc quantities, with all remaining components set to zero.

\section{Results}

\subsection{Experimental System}

The left photo in Figure 7 shows the interior part of the experimental system, where the IGBT modules (IFS150V12PT4) and the dc link are visible in the center. One IGBT module was used in the experiments as a VSI and the second module was switched off and used as a diode bridge rectifier.

The VSI control was implemented on a system-on-chip unit Xilinx Zynq 7000. It contains an FPGA and an ARM Cortex- $A 9$ double-core processor. The FPGA comprises the measurement data collection from the $\Delta \Sigma$ modulators Analog Devices AD7401A. The digital filters (decimators) were implemented as Sinc3 filters and were implemented on the FPGA. The FPGA also incorporates the low-level control of the power converters, whereas the high level control was implemented in the processor.
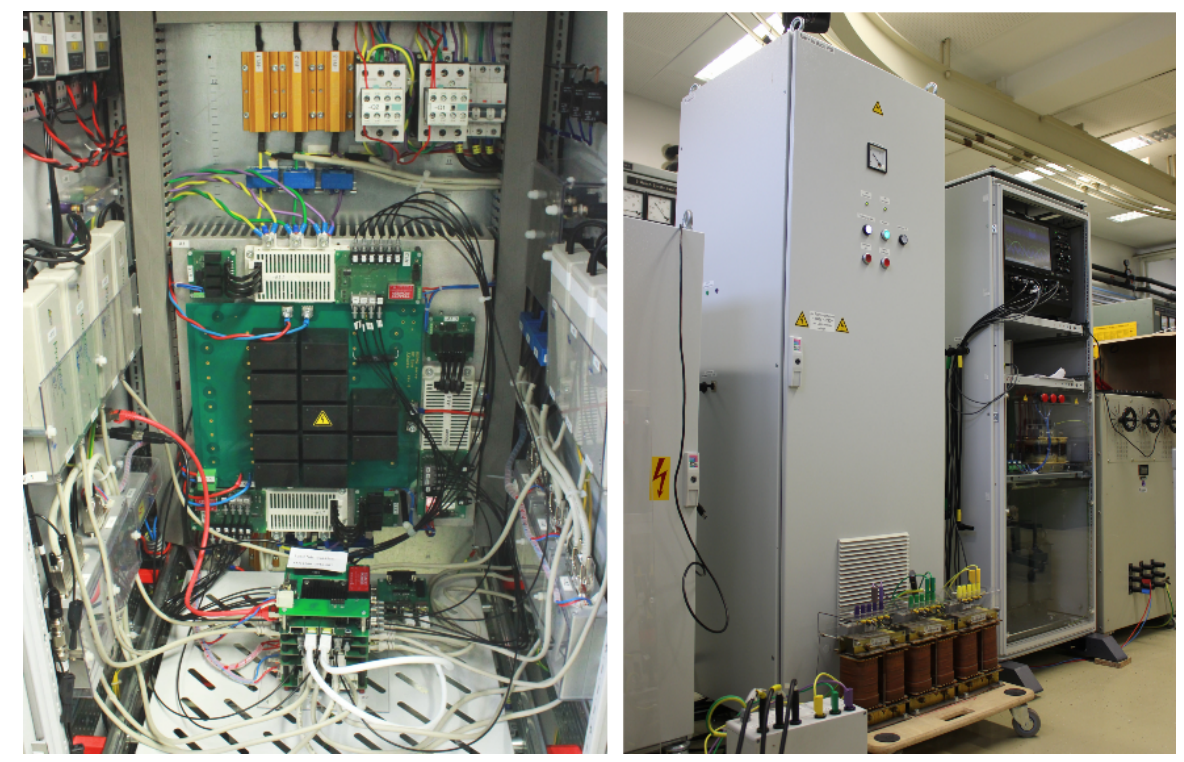

Figure 7. Photos of the experimental system. 
The voltage differential probe measuring the dc-link voltage (Tektronix P5200) and the line-to-line voltage (Testec TT-SI-9001) were connected directly to the terminals of the IGBT module. The ac-side current was measured in phase 1 using a current probe (Agilent N2782B). The dc-side current and the rectifier current were measured with Rogowski coils (PEM CWTO3LF), thus the dc component was excluded in the presented results. The gate signals of the top and bottom IGBTs of phase 1 were measured at the input terminals of the gate driver (Testec TT-SI-9001).

The data acquisition was performed using an eight channel oscilloscope Teledyne Lecroy HDO8108, which is depicted on the right side of Figure 7 . By using a high sampling rate of $250 \mathrm{MS} / \mathrm{s}, 20 \mathrm{MHz}$ analog input filters for the channels, and a 12 bit quantization, a good time and amplitude resolution of the measured waveforms was provided. The measured waveforms were postprocessed in Matlab to calculate the spectra.

\subsection{Results for AD-PWM Model}

To validate the correct derivation of (26), the equation was numerically evaluated for an example case with a duty cycle spectrum that contains frequency components at the fundamental frequency and at the fifth harmonic. The equivalent time-domain waveform is

$$
x(t)=0.5 \cdot \cos \left(2 \pi f_{0} t\right)+0.5 \cdot \cos \left(2 \pi \cdot 5 f_{0} t\right)
$$

with a fundamental frequency of $f_{0}=50 \mathrm{~Hz}$. The sampling frequency of the $\mathrm{ZOH}$ is $f_{\mathrm{s}}=4 \mathrm{kHz}$ and the carrier frequency is $f_{\mathrm{sw}}=2 \mathrm{kHz}$. The first graph of Figure 8 shows the amplitude spectrum resulting from the application of (26) for the first 100 harmonics of $f_{0}$. The indexes of summation in the evaluation of (26) were limited to $-m_{\max } \leq m \leq m_{\max }$ and $1 \leq n \leq n_{\max }$, with $m_{\max }=3$ and $n_{\max }=15$.
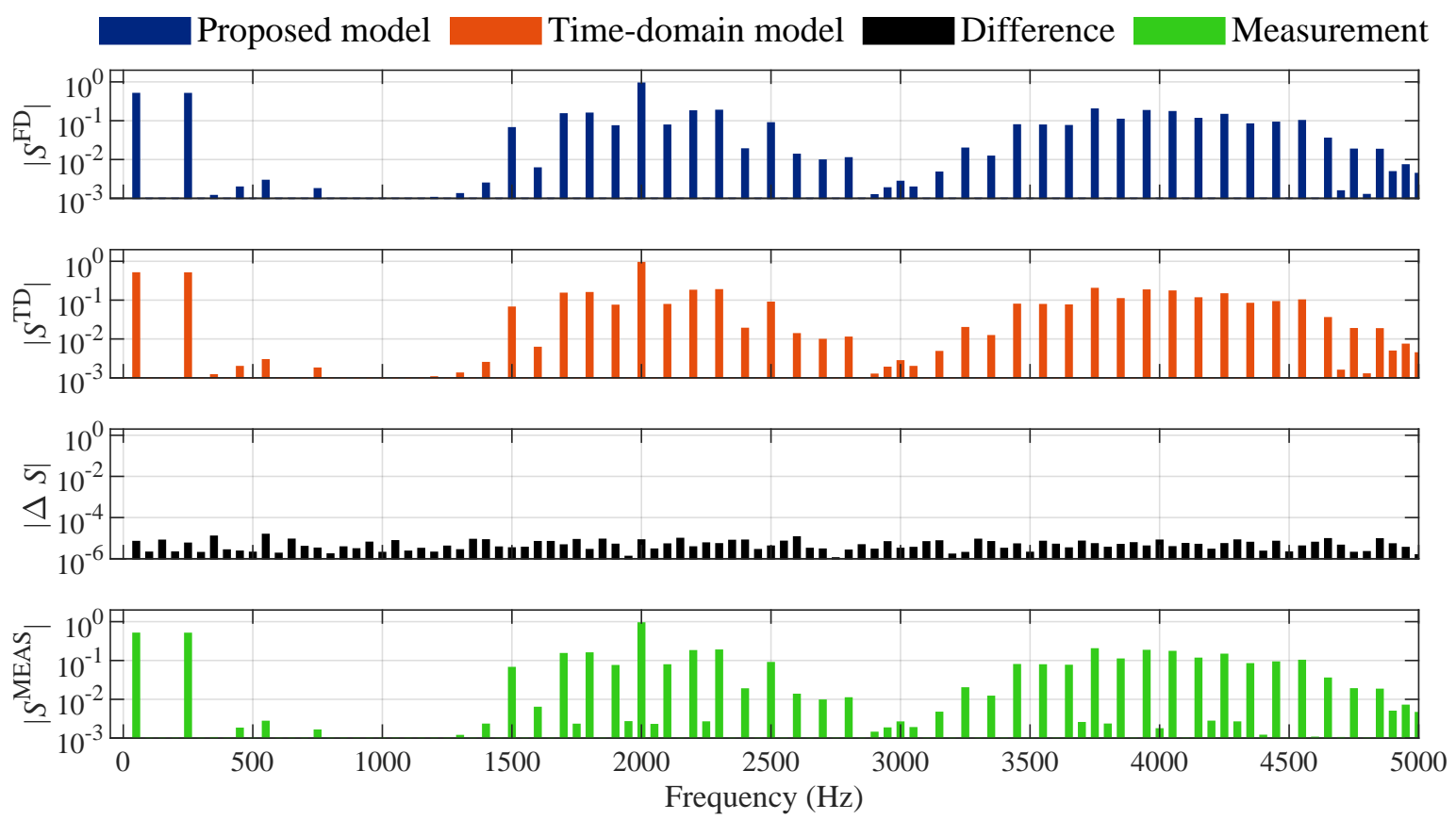

Figure 8. Amplitude spectra of the proposed frequency-domain model (first graph), the time-domain simulation (second graph), their difference based on the complex spectra (third graph), and the amplitude spectra from measurement results (fourth graph). 
The second graph shows the spectrum from a time-domain simulation in Simulink, resulting from a Fast Fourier transform (FFT) of the results calculated in Matlab. The third graph depicts the difference of spectra with black bars, with

$$
|\Delta S(f)|=\left|S^{\mathrm{TD}}(f)-S^{\mathrm{FD}}(f)\right|,
$$

where TD and FD identify the time-domain simulation and the frequency-domain calculation, respectively. The differences in the spectra are much lower than $10^{-3}$. Because the spectral difference is shown for the complex spectra, the validation also ensures that the phase information is correct. The fourth graph of Figure 8 presents the amplitude spectra $\left|S^{\operatorname{MEAS}}(f)\right|$ resulting from measurement of the gate-driver signals, further validating the proposed model.

\subsection{Results for AD-PWM Model with DTFT Input}

A numerical comparison of the two models presented in Figure 6a,b was performed, using the model by Song and Sarwate ([11], (61)) for ND-PWM and the proposed model for AD-PWM in (26). The input is the spectrum of the sampled duty cycle, as shown in Figure 9. It comprises a fundamental frequency component with an amplitude of $\left|D_{, 1}\right|=0.7$ and a seventh harmonic component with an amplitude of $\left|D_{, 7}\right|=0.1$. The carrier to fundamental ratio is $f_{\mathrm{sw}} / f_{d 0}=3000 / 50=60$.

The amplitude spectrum of the switching function is shown in the graphs on the left side of Figure 10, while varying the parameters $m_{\max }$ and $n_{\max }$. The first graph compares the frequency-domain model with a time-domain model for $m_{\max }=1, n_{\max }=7, k_{\max }=150$. Large deviations between the complex spectra are visible in the second graph. Increasing $m_{\max }$ and $n_{\max }$ leads only to a minor reduction of the deviations, because of the low number of harmonics $k_{\max }$ considered in the duty-cycle spectrum (third and fourth graph). With these parameters, the second and third sideband groups depicted in Figure 9 are neglected, resulting in a faulty calculation of the first carrier group for the switching function spectrum.

The method demonstrated in Figure $6 \mathrm{~b}$ converts the DTFT of the duty-cycle first into a CTFT, removing the high-frequency components shown in Figure 9 before application to the AD-PWM model. The right side of Figure 10 presents the evaluation of this model for the same parameters as for the ND-PWM model. Even though the modeling approach seems to be more complex, a high accuracy is reached for relatively low numbers of $k_{\max }$. Due to the rectangular filter, the duty-cycle components requiring consideration in the PWM model are limited to $f_{\mathrm{c}} / 2$. This numerical example demonstrates that model $b$ ) requires a lower number of harmonics $k_{\max }$ in the duty-cycle to reach the same accuracy as the AD-PWM model, which is crucial for the numerical solution process of the overall system.

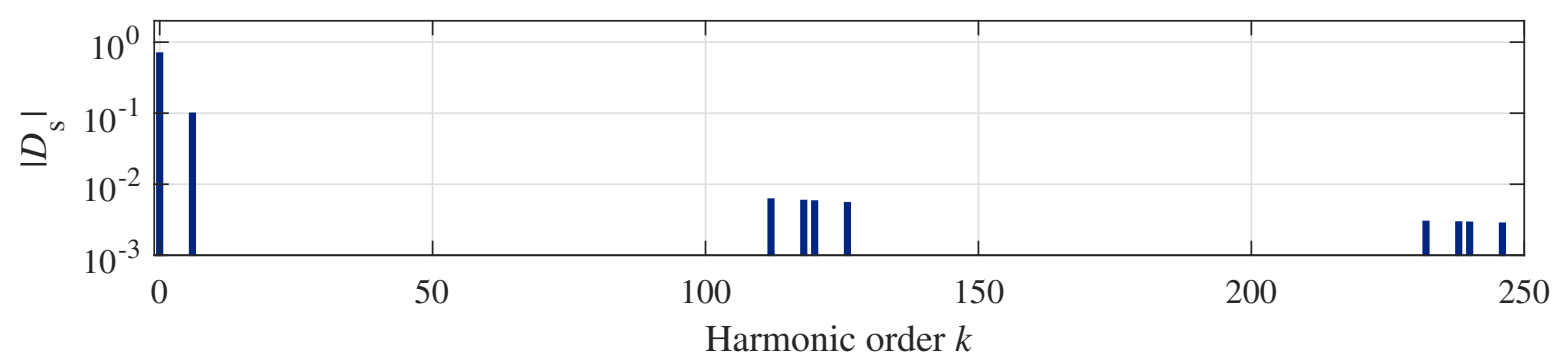

Figure 9. Spectrum of the sampled and held duty cycle with two components at $k=1$ and $k=7$. Due to sampling, components around multiples of the $f_{\mathrm{c}} / f_{0}=60$ are present [27]. 


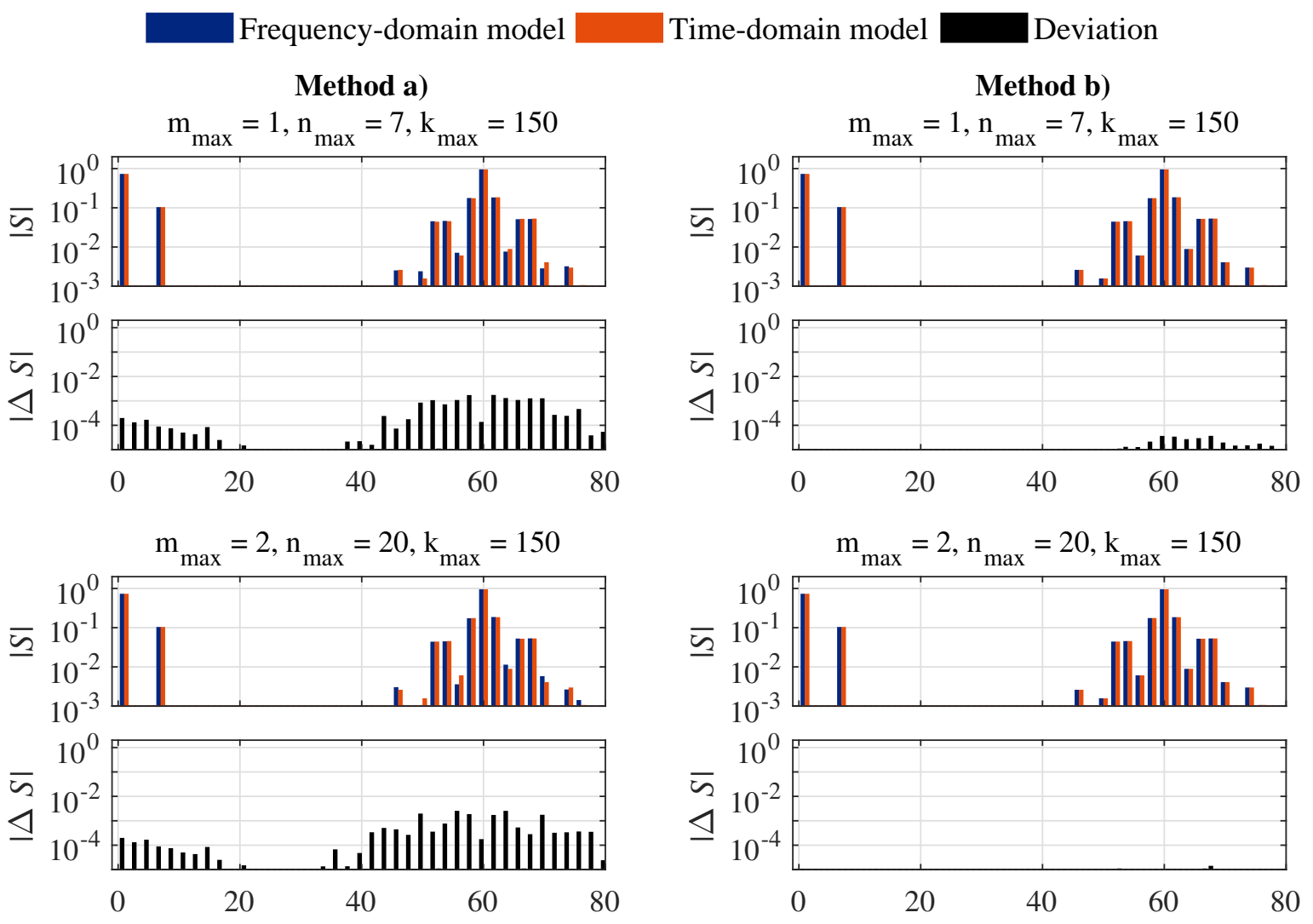

Figure 10. Evaluation of the models depicted in Figure 6a (left graphs) and Figure $6 \mathrm{~b}$ (right graphs).

The parameters $m_{\max }$ and $n_{\max }$ are varied from top to bottom [27].

\subsection{Results for the VSI Model}

The evaluation of the VSI model was conducted for an example case with the parameters listed in Table 1. The PI controller was designed using the technical optimum. Harmonic components were introduced by the nonreactive dc current source with frequencies of $n \cdot 6 \cdot f_{\mathrm{g}}, n \in \mathbb{N}$ as an example of a three-phase diode rectifier fed from a symmetrical $400 \mathrm{~V}$ grid.

The frequency-domain model was evaluated with $k_{\max }=70, m_{\max }=1$, and $n_{\max }=7$. The magnitude spectra are depicted in Figure 11, comparing the results from the frequency-domain model to results from a time-domain simulation. As shown in Figure 1, the spectrum $I_{\text {rec }}$ and the dc-component of the dc-link voltage in the frequency-domain model are input signals of the frequency-domain model and were selected to be the same values as in the time-domain simulation. Low-frequency components were introduced into the duty cycle spectrum through the control loop of the ac-side currents and the feedback of the dc-link voltage. These components are a product of the disturbance current of the diode rectifier, which are propagated to the sensed dc-link voltage and the sensed ac-side currents. Because the PWM process is nonlinear, the harmonic components of the duty cycle spectrum create additional base-band and side-band harmonics in the switching function spectrum.

The results of the two models have a high rate of conformity, which is demonstrated by the low deviations of the complex spectra shown in Figure 12. Minor deviations visible around the switching frequency can be explained by the low number of considered harmonics, as demonstrated by the previous examination shown in Figure 10. For instance, the components in the duty cycle spectrum around the first carrier-sideband group would cancel out with components of the second carrier-sideband group, indicating that an improvement of the method to enable a higher number of considered harmonics is desirable. 
A comparison of the frequency-domain results with measurement results is presented in Figure 13, confirming the pattern of the calculated spectra. The VSI was fed from a three-phase diode rectifier and the spectrum $I_{\text {rec }}$ and the dc-component of the dc-link voltage were used as input signals for the frequency-domain model. The deviations between the frequency-domain model and measurements are higher in comparison to the deviations from the simulation results, indicating the influence of neglected effects in the models such as converter dead time.

Table 1. Parameters of the example system.

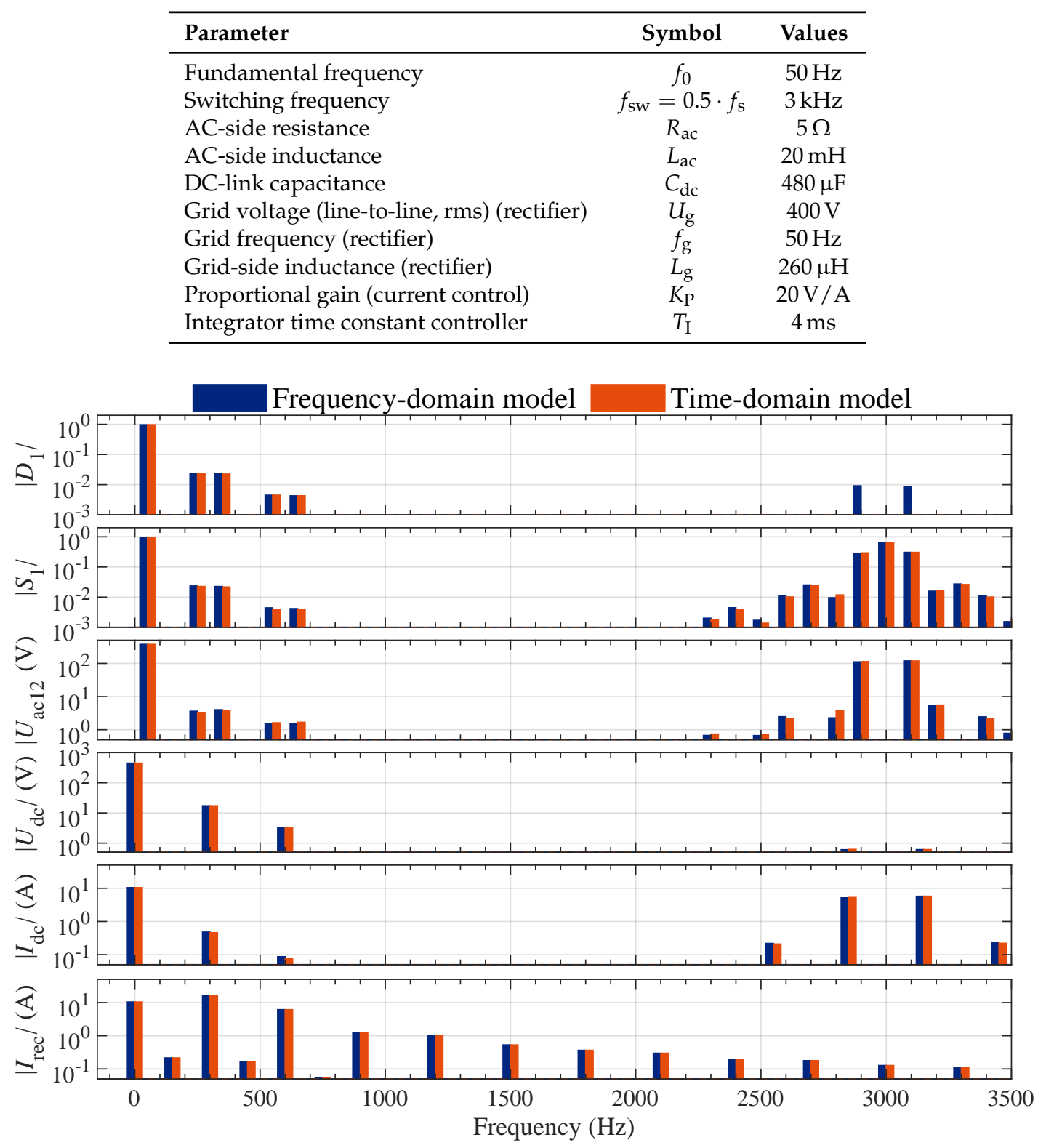

Figure 11. Comparison of the results for closed-loop control using the frequency-domain model (blue) and the time-domain model (red). 


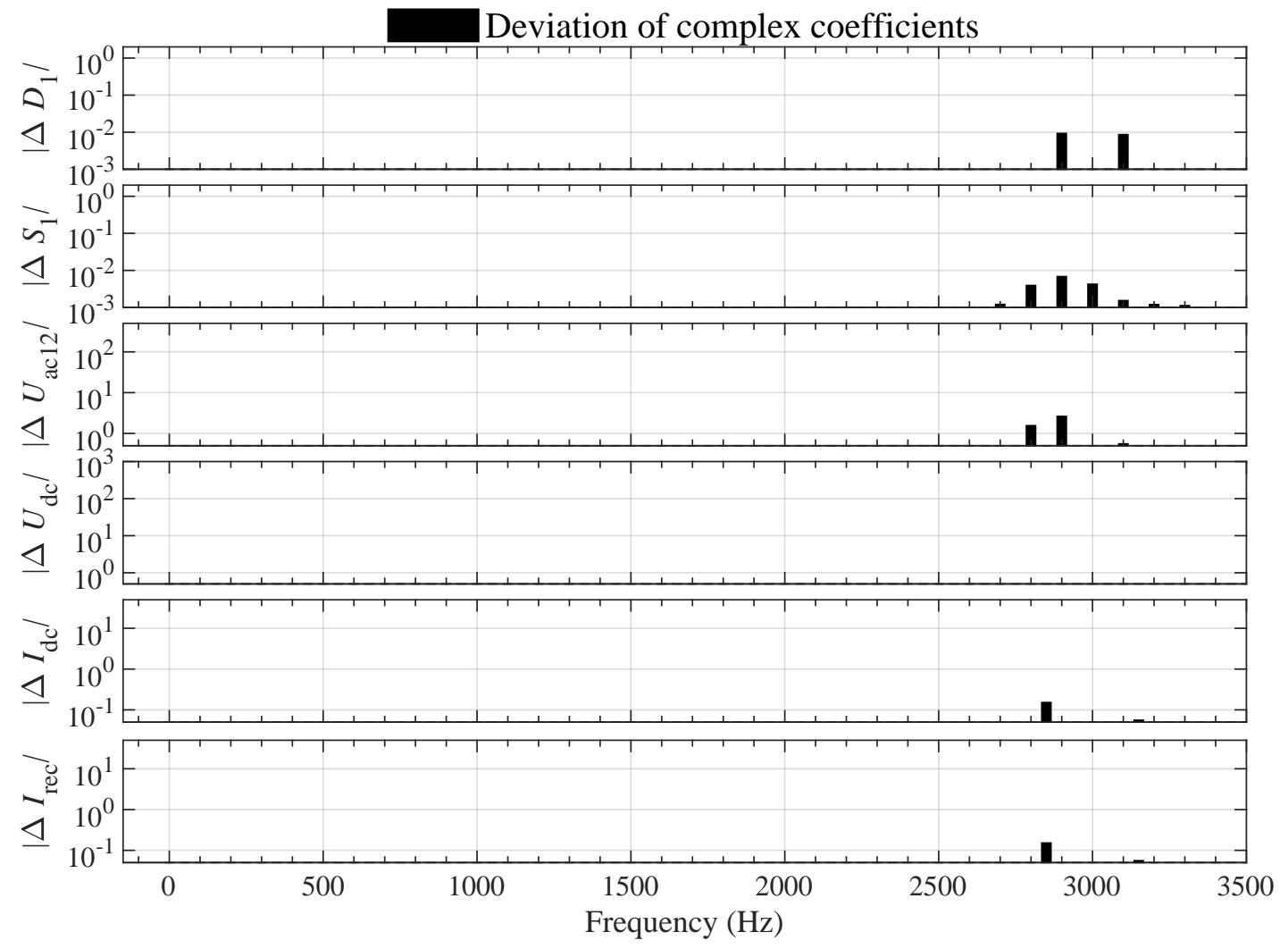

Figure 12. Deviation of the complex spectra of the frequency-domain model from the time-domain model presented in Figure 11 for closed-loop control.

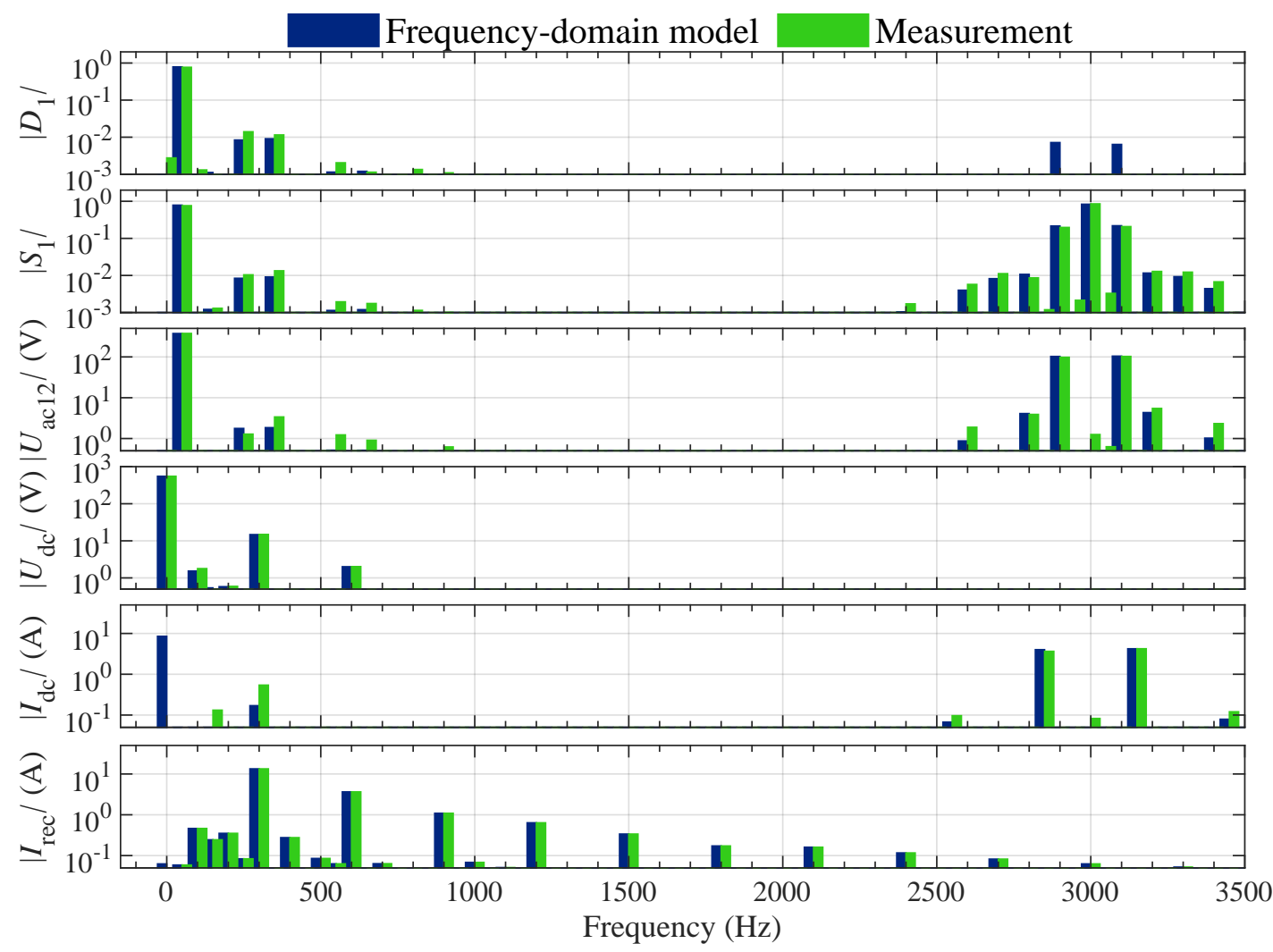

Figure 13. Comparison of frequency-domain model and measurements for a three-phase VSI with closed-loop control for Case I. 


\section{Conclusions}

The control PWM signals and the electrical system of a VSI form a closed-loop system. A system model was developed in the frequency-domain that incorporates the interaction of the closed-loop control, the PWM process, and the power electronic devices as ideal switches. Although past methods in the literature demonstrate unidirectional descriptions for the individual parts of the converter system, the approach posed here includes their mutual dependencies and nonlinear interactions. The overall nonlinear equation system was numerically and simultaneously solved. The analysis showed how the propagation of harmonic components through the control loop alters the mutually coupled spectra of the duty cycle and the switching function.

A PWM model that considers multiple-frequency duty cycles is necessary to cover these interactions. This paper demonstrates the derivation of a revised analytic frequency-domain model for AD-PWM that is valid for multiple-frequency input signals. Low deviations between the numerical results from this model and those of time-domain simulations, as well as those of measurements, support the proposed equations. This paper illustrated the approximation of a regularly-sampled PWM model with a continuous-time input signal by a naturally-sampled PWM model that is extended with a sample-and-hold unit at its input. For the case of discrete-time input signals, an inverse sample-and-hold process in combination with a regularly-sampled PWM can be applied. It was demonstrated that a smaller number of considered harmonics is required for this approach to achieve the same accuracy as a naturally-sampled PWM model.

Application of the presented method is recommended when the interaction of the harmonic components cannot be neglected and when the base band harmonics and the switching band harmonics cannot be clearly separated. Due to the detailed system description, the computational effort is generally high and increases further with the number of considered harmonics and the number of system variables. An enhancement of the computational speed is desirable, when large numbers of considered harmonics and multiple nonlinear effects are present. For this, further development of the system description and an extensive study of solvers and an analytical Jacobian matrix are recommended.

Author Contributions: Conceptualization, methodology, validation, formal analysis, writing-original draft preparation, M.J.; funding acquisition, project administration, supervision, writing-review and editing, A.M.; All authors have read and agreed to the published version of the manuscript.

Funding: This research was funded in part by the Volkswagen Foundation's grant "Niedersächsisches Vorab 2014" within the project AMSES (Aggregated Models for the Simulation of Electromechanical Power Systems). The publication of this article was funded by the Open Access Fund of Leibniz Universität Hannover.

Conflicts of Interest: The authors declare no conflict of interest.

\section{Abbreviations}

The following abbreviations are used in this manuscript:

$\begin{array}{ll}\text { ADC } & \text { Analog-to-digital converter } \\ \text { AD-PWM } & \text { Asymmetrical regularly-sampled double-edge PWM } \\ \text { CTFT } & \text { Continuous-time Fourier transform } \\ \text { DTFT } & \text { Discrete-time Fourier transform } \\ \text { FFT } & \text { Fast Fourier transform } \\ \text { LE } & \text { Leading edge } \\ \text { LE-PWM } & \text { Leading-edge PWM } \\ \text { ND-PWM } & \text { Naturally-sampled double-edge PWM } \\ \text { PWM } & \text { Pulse-width modulation } \\ \text { TE } & \text { Trailing edge } \\ \text { TE-PWM } & \text { Trailing-edge PWM } \\ \text { VSI } & \text { Voltage-source inverter } \\ \text { ZOH } & \text { Zero-order hold }\end{array}$




\section{Appendix A}

This appendix provides the derivation of a frequency-domain expression for AD-PWM in (26) as a revision of the model presented in [11]. When using double-edge PWM, the placement of both edges within the switching period depends on the duty cycle. Sampling the duty cycle at the beginning and the center of the carrier period results in AD-PWM. Its analysis can be simplified by regarding the rising edge as a trailing-edge PWM (TE-PWM) signal and the falling edge as a leading-edge PWM (LE-PWM) signal [31]. This approach is illustrated in Figure A1, where the AD-PWM process is depicted for one switching period and a positive duty cycle. Subfigure a shows the comparison of a sampled duty cycle (red dashed line) with a triangular carrier signal (black solid line). The duty cycle is sampled and held at the beginning of the switching period for the leading edge (LE) and at the center of the period for the trailing edge (TE). The resulting output can be composed of:

(1) A rectangular signal $s_{\mathrm{c}}$ with constant duty cycle of $50 \%$ (Subfigure d): In accordance with Song and Sarwate [11], the signal $s_{\mathcal{C}}(t)$ is defined as a rectangular wave

$$
s_{\mathrm{c}}(t)=\left\{\begin{array}{ccc}
1 & \text { for } & m T_{\mathrm{Sw}} \leq t<(m+1 / 2) T_{\mathrm{sw}} \\
-1 & \text { for } & (m+1 / 2) T_{\mathrm{sw}} \leq t<(m+1) T_{\mathrm{sw}}
\end{array},\right.
$$

where $m \in \mathbb{Z}, T_{\mathrm{sw}}=1 / f_{\mathrm{sw}}$, and $t$ is the time. The signal in (Subfigure $\mathrm{d}$ ) is right-shifted by $T_{\mathrm{sw}} / 4$.

(2) A rectangular signal $s_{\mathrm{dLE}}$ that is determined by the LE (Subfigure e): Its rising edge results from a comparison of the duty-cycle sampled at the beginning of the switching period $d\left[m T_{\mathrm{sw}}\right] / 2$ with a LE carrier signal (Subfigure b). The original duty cycle is plotted in light red and the halved value is depicted in red. The carrier signal is left-shifted by $T_{\mathrm{sW}} / 4$ (original carrier depicted in gray and shifted carrier in black). The falling edge of $s_{\mathrm{dLE}}$ is fixed to $T_{\mathrm{sW}} / 4+m T_{\mathrm{sw}}$.

(3) A rectangular signal $s_{\mathrm{dTE}}$ that is determined by the TE (Subfigure $\mathrm{f}$ ): Its falling edge results from a comparison of the duty-cycle sampled at the center of the switching period $d\left[(m+1 / 2) T_{\mathrm{sw}}\right] / 2$ with a TE carrier signal that is right-shifted by $T_{\mathrm{SW}} / 4$ (Subfigure c). The rising edge of $s_{\mathrm{dTE}}$ is fixed to $3 T_{\mathrm{sw}} / 4+m T_{\mathrm{sw}}$.

Their superposition yields the switching function for AD-PWM, with

$$
\begin{aligned}
s_{\mathrm{AD}}(t, d)=s_{\mathrm{C}}\left(t-T_{\mathrm{SW}} / 4\right) & +s_{\mathrm{dLE}}\left(t+T_{\mathrm{SW}} / 4 ; d\left[m T_{\mathrm{SW}}\right] / 2\right) \ldots \\
& +s_{\mathrm{dTE}}\left(t-T_{\mathrm{SW}} / 4 ; d\left[(m+1 / 2) T_{\mathrm{SW}}\right] / 2\right) .
\end{aligned}
$$

The sampling points of the duty cycle $\left[m T_{\mathrm{sw}}\right]$ and $\left[(m+1 / 2) T_{\mathrm{sw}}\right]$ are included to stress the difference between the TE and the LE. This equation differs from the result of Song and Sarwate ([11], (56)), where the different sampling instances for LE and TE are not represented. The Fourier transform of the switching function in (A2) results in

$$
\begin{aligned}
S_{\mathrm{AD}}(f, d)=\mathrm{e}^{-\mathrm{j} \pi f T_{\mathrm{sw}} / 2} \cdot S_{\mathrm{c}}(f) & +\mathrm{e}^{-\mathrm{j} \pi f T_{\mathrm{sw}} / 2} \cdot S_{\mathrm{dTE}}\left(f, d\left[(m+1 / 2) T_{\mathrm{sW}}\right] / 2\right) \ldots \\
& +\mathrm{e}^{-\mathrm{j} \pi f T_{\mathrm{sw}} / 2} \cdot S_{\mathrm{dLE}}\left(f, d\left[m T_{\mathrm{sW}}\right] / 2\right) .
\end{aligned}
$$

In the following equations, explicit frequency-domain expressions of the three components in (A3) are derived. The spectrum of the rectangular function of constant duty cycle is given in (24). 


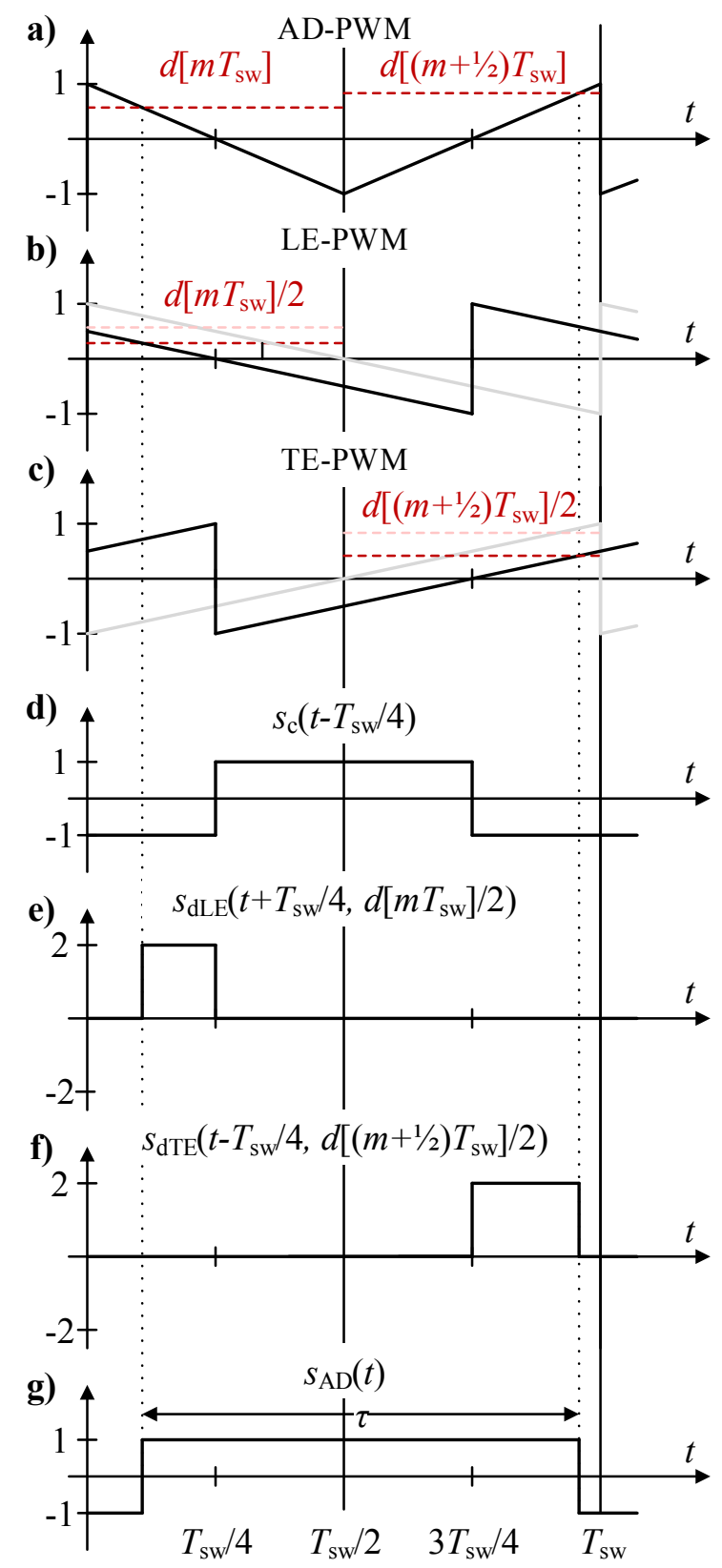

Figure A1. Derivation of switching function for AD-PWM: (a) Comparison of an AD-PWM carrier signal (black line) with a sampled duty cycle sampled at $m T_{\mathrm{Sw}}$ and $(m+1 / 2) T_{\mathrm{SW}}$ (red dashed line). (b) Equivalent description for the leading edge using a LE-PWM carrier signal left-shifted by $T_{\mathrm{sw}} / 4$. The duty cycle's value is halved and sampled at $m T_{\mathrm{sw}}$. (c) Equivalent description for the trailing edge using a TE-PWM carrier signal right-shifted by $T_{\mathrm{Sw}} / 4$. The duty cycle's value is halved and sampled at $(m+1 / 2) T_{\mathrm{sw}}$. (d) Rectangular function with a duty cycle of $50 \%$ right-shifted by $T_{\mathrm{SW}} / 4$. (e) Duty-cycle-dependent part for LE resulting from (b). (f) Duty-cycle-dependent part for TE resulting from (c). (g) Resulting switching function comprising (d) + (e) + (f) [27].

The two duty-cycle-dependent components can be expressed by using a Lagrange extension of the rectangular functions, where the TE component can be expressed as

$$
\begin{aligned}
S_{\mathrm{dTE}}(f, d)= & \frac{1}{\mathrm{j} \pi f} \cdot \mathrm{e}^{-\mathrm{j} \pi f T_{\mathrm{sW}}} \cdot \sum_{n=1}^{\infty}-\frac{\left(-\mathrm{j} \pi f T_{\mathrm{sW}}\right)^{n}}{n !} \cdot \sum_{m=-\infty}^{\infty}\left(d\left[m T_{\mathrm{sW}}\right]\right)^{n} \cdot \mathrm{e}^{-\mathrm{j} 2 \pi f m T_{\mathrm{sW}}}, \\
& k \in \mathbb{Z}, n \in \mathbb{N} .
\end{aligned}
$$


Similarly, the LE component results in

$$
\begin{aligned}
S_{\mathrm{dLE}}(f, d) & =-S_{\mathrm{dTE}}(f,-d) \\
& =-\frac{1}{\mathrm{j} \pi f} \cdot \mathrm{e}^{-\mathrm{j} \pi f T_{\mathrm{sw}}} \cdot \sum_{n=1}^{\infty}-\frac{\left(\mathrm{j} \pi f T_{\mathrm{sW}}\right)^{n}}{n !} \cdot \sum_{m=-\infty}^{\infty}\left(d\left[m T_{\mathrm{sW}}\right]\right)^{n} \cdot \mathrm{e}^{-\mathrm{j} 2 \pi f m T_{\mathrm{sw}}} .
\end{aligned}
$$

In order to express the switching function spectrum as a function of the duty cycle spectrum $D(f) \longmapsto S_{\mathrm{AD}}(f)$, the time-domain expression $\left(d\left[m T_{\mathrm{sw}}\right]\right)^{n}$ needs to be replaced by a frequency-domain expression. By acknowledging that exponentiation of a signal in the time domain relates to a repeated convolution in the frequency domain, the Fourier transform of the continuous-time duty-cycle is written as $D^{* n}(f)$, see (25). Because the duty-cycle for regularly-sampled PWM is a discrete-time signal, the discrete-time Fourier transform is applied, which results in

$$
\sum_{m=-\infty}^{\infty}\left(d\left[m T_{\mathrm{sw}}\right]\right)^{n} \cdot \mathrm{e}^{-\mathrm{j} 2 \pi f m T_{\mathrm{sw}}}=f_{\mathrm{sw}} \cdot \sum_{m=-\infty}^{\infty} D^{* n}\left(f-m f_{\mathrm{sw}}\right) .
$$

The influence of the duty cycle's time shift of $T_{\mathrm{sw}} / 2$ on the Fourier transform in (A7) yields

$$
\sum_{m=-\infty}^{\infty}\left(d\left[(m+1 / 2) T_{\mathrm{sw}}\right]\right)^{n} \cdot \mathrm{e}^{-\mathrm{j} 2 \pi f m T_{\mathrm{sw}}}=f_{\mathrm{sw}} \cdot \sum_{m=-\infty}^{\infty} \mathrm{e}^{\mathrm{j} \pi \cdot\left(f-m f_{\mathrm{sw}}\right) T} \cdot D^{* n}\left(f-m f_{\mathrm{sW}}\right) .
$$

Its application to (A4) allows for the description of the TE component for AD-PWM, with

$$
\begin{aligned}
S_{\mathrm{dTE}}\left(f, d\left[(m+1 / 2) T_{\mathrm{sw}}\right] / 2\right) \\
\quad=\frac{1}{\mathrm{j} \pi f} \cdot \mathrm{e}^{-\mathrm{j} \pi f T_{\mathrm{sw}}} \cdot \sum_{n=1}^{\infty}-\frac{\left(-\mathrm{j} \pi f T_{\mathrm{sW}}\right)^{n}}{n !} \cdot \sum_{m=-\infty}^{\infty}\left(d\left[(m+1 / 2) T_{\mathrm{sW}}\right] / 2\right)^{n} \cdot \mathrm{e}^{-\mathrm{j} 2 \pi f m T_{\mathrm{sw}}} \\
\quad=\mathrm{e}^{-\mathrm{j} \pi f T_{\mathrm{sw}}} \cdot \sum_{m=-\infty}^{\infty} \sum_{n=1}^{\infty} \frac{\left(-\mathrm{j} \pi f T_{\mathrm{sW}}\right)^{n-1}}{2^{n} \cdot n !} \cdot D^{* n}\left(f-m f_{\mathrm{sw}}\right) \cdot \mathrm{e}^{\mathrm{j} \pi \cdot\left(f-m f_{\mathrm{sw}}\right) T_{\mathrm{sw}}} .
\end{aligned}
$$

Similarly, the LE component is expressed as

$$
\begin{aligned}
S_{\mathrm{dLE}} & \left(f, d\left[m T_{\mathrm{sW}}\right] / 2\right) \\
& =-\frac{1}{\mathrm{j} \pi f} \cdot \mathrm{e}^{-\mathrm{j} \pi f T_{\mathrm{sw}}} \cdot \sum_{n=1}^{\infty}-\frac{\left(\mathrm{j} \pi f T_{\mathrm{sW}}\right)^{n}}{n !} \cdot \sum_{m=-\infty}^{\infty}\left(d\left[k T_{\mathrm{sW}}\right] / 2\right)^{n} \cdot \mathrm{e}^{-\mathrm{j} 2 \pi f m T_{\mathrm{sw}}} \\
& =\mathrm{e}^{-\mathrm{j} \pi f T_{\mathrm{sw}}} \cdot \sum_{m=-\infty}^{\infty} \sum_{n=1}^{\infty} \frac{\left(\mathrm{j} \pi f T_{\mathrm{sW}}\right)^{n-1}}{2^{n} \cdot n !} \cdot D^{* n}\left(f-m f_{\mathrm{sW}}\right) .
\end{aligned}
$$

Applying the results from (A9) and (A10) to (A3) results in

$$
\begin{aligned}
& S_{\mathrm{AD}}(f, D)=\mathrm{e}^{-\mathrm{j} \pi f T_{\mathrm{sw}} / 2} \cdot S_{\mathrm{c}}(f) \ldots \\
& +\mathrm{e}^{-\mathrm{j} \pi f T_{\mathrm{sw}} / 2} \cdot \mathrm{e}^{-\mathrm{j} \pi f T_{\mathrm{sw}}} \cdot \sum_{m=-\infty}^{\infty} \sum_{n=1}^{\infty} \frac{\left(-\mathrm{j} \pi f T_{\mathrm{sW}}\right)^{n-1}}{2^{n} \cdot n !} \cdot D^{* n}\left(f-m f_{\mathrm{sw}}\right) \cdot \mathrm{e}^{\mathrm{j} \pi \cdot\left(f-m f_{\mathrm{sw}}\right) T} \ldots \\
& +\mathrm{e}^{+\mathrm{j} \pi f T_{\mathrm{sw}} / 2} \cdot \mathrm{e}^{-\mathrm{j} \pi f T_{\mathrm{sw}}} \cdot \sum_{m=-\infty}^{\infty} \sum_{n=1}^{\infty} \frac{\left(\mathrm{j} \pi f T_{\mathrm{sw}}\right)^{n-1}}{2^{n} \cdot n !} \cdot D^{* n}\left(f-m f_{\mathrm{sw}}\right),
\end{aligned}
$$

which can be summarized in the final form of (26).

\section{References}

1. Bierhoff, M.; Fuchs, F. DC-Link Harmonics of Three-Phase Voltage-Source Converters Influenced by the Pulsewidth-Modulation Strategy-An Analysis. IEEE Trans. Ind. Electron. 2008, 55, 2085-2092. [CrossRef] 
2. McGrath, B.; Holmes, D. A General Analytical Method for Calculating Inverter DC-Link Current Harmonics. IEEE Trans. Ind. Appl. 2009, 45, 1851-1859. [CrossRef]

3. Meyer, R.; Zlotnik, A.; Mertens, A. Fault Ride-Through Control of Medium-Voltage Converters With LCL Filter in Distributed Generation Systems. IEEE Trans. Ind. Appl. 2014, 50, 3448-3456. [CrossRef]

4. Fuchs, F. Converter Control for Wind Turbines when Operating inWeak Grids Containing Resonances. Ph.D. Thesis, Leibniz Universität Hannover, Hanover, Germany, 2017.

5. Odavic, M.; Sumner, M.; Zanchetta, P.; Clare, J.C. A Theoretical Analysis of the Harmonic Content of PWM Waveforms for Multiple-Frequency Modulators. IEEE Trans. Power Electron. 2010, 25, 131-141. [CrossRef]

6. Rahmani, S.; Al-Haddad, K.; Kanaan, H.Y. Two PWM techniques for single-phase shunt active power filters employing a direct current control strategy. IET Power Electron. 2008, 1, 376-385. [CrossRef]

7. Bowes, S.; Bird, B. Novel approach to the analysis and synthesis of modulation processes in power convertors. Proc. Inst. Electr. Eng. 1975, 122, 507-513. [CrossRef]

8. Holmes, G. A Generalised Approach to the Modulation and Control of Hard Switched Converters. Ph.D. Thesis, Monash University, Melbourne, Australia, 1997.

9. Holmes, G.; Lipo, T.A. Pulse Width Modulation for Power Converters: Principles and Practice; Wiley-IEEE Press: Hoboken, NJ, USA, 2003.

10. Cox, S.M. Voltage and current spectra for a single-phase voltage source inverter. IMA J. Appl. Math. 2009, 74, 782-805. [CrossRef]

11. Song, Z.; Sarwate, D.V. The frequency spectrum of pulse width modulated signals. Signal Process. 2003, 83, 2227-2258. [CrossRef]

12. Kostic, D.J.; Avramovic, Z.Z.; Ciric, N.T. A New Approach to Theoretical Analysis of Harmonic Content of PWM Waveforms of Single- and Multiple-Frequency Modulators. IEEE Trans. Power Electron. 2013, 28, 4557-4567. [CrossRef]

13. Mouton, H.D.T.; McGrath, B.; Holmes, D.G.; Wilkinson, R.H. One-Dimensional Spectral Analysis of Complex PWM Waveforms Using Superposition. IEEE Trans. Power Electron. 2014, 29, 6762-6778. [CrossRef]

14. Thakur, S.S.; Odavic, M.; Allu, A.; Zhu, Z.Q.; Atallah, K. Theoretical Harmonic Spectra of PWM Waveforms Including DC Bus Voltage Ripple-Application to a Low-Capacitance Modular Multilevel Converter. IEEE Trans. Power Electron. 2020, 35, 9291-9305. [CrossRef]

15. Milovanović, S.; Dujić, D. Comprehensive Spectral Analysis of PWM Waveforms With Compensated DC-Link Oscillations. IEEE Trans. Power Electron. 2020, 35, 12898-12908. [CrossRef]

16. Wester, G.W.; Middlebrook, R.D. Low-Frequency Characterization of Switched dc-dc Converters. IEEE Trans. Aerosp. Electron. Syst. 1973, AES-9, 376-385. [CrossRef]

17. Cuk, S. Modelling, Analysis, and Design of Switching Converters. Ph.D. Thesis, California Institute of Technology, Pasadena, CA, USA, 1977.

18. Middlebrook, R.D.; Ćuk, S. A general unified approach to modelling switching-converter power stages. Int. J. Electron. 1977, 42, 521-550. [CrossRef]

19. Erickson, R.W.; Maksimovic, D. Fundamentals of Power Electronics, 2nd ed.; Springer US: New York, NY, USA, 2001.

20. Maksimovic, D.; Stankovic, A.M.; Thottuvelil, V.J.; Verghese, G.C. Modeling and simulation of power electronic converters. Proc. IEEE 2001, 89, 898-912. [CrossRef]

21. Maksimovic, D.; Zane, R. Small-Signal Discrete-Time Modeling of Digitally Controlled PWM Converters. IEEE Trans. Power Electron. 2007, 22, 2552-2556. [CrossRef]

22. Hiti, S. Modeling and Control of Three-Phase PWM Converters. Ph.D. Thesis, Virginia Tech, Blacksburg, VA, USA, 1995.

23. Sun, J. Small-Signal Methods for AC Distributed Power Systems-A Review. IEEE Trans. Power Electron. 2009, 24, 2545-2554. [CrossRef]

24. Corradini, L.; Maksimovic, D.; Mattavelli, P.; Zane, R. Digital Control of High-Frequency Switched-Mode Power Converters; Wiley-IEEE Press: Hoboken, NJ, USA, 2015.

25. Almér, S.; Jönsson, U. Harmonic analysis of pulse-width modulated systems. Automatica 2009, 45, 851-862. [CrossRef]

26. Yue, X.; Boroyevich, D.; Lee, F.C.; Chen, F.; Burgos, R.; Zhuo, F. Beat Frequency Oscillation Analysis for Power Electronic Converters in DC Nanogrid Based on Crossed Frequency Output Impedance Matrix Model. IEEE Trans. Power Electron. 2018, 33, 3052-3064. [CrossRef] 
27. John, M. Frequency-Domain Modeling of Harmonic Interactions in Pulse-Width Modulated Voltage-Source Inverter Drives. Ph.D. Thesis, Leibniz Universität Hannover, Hanover, Germany, 2019.

28. Candy, J.C.; Tmes, G.C. Oversampling Delta-Sigma Data Converters: Theory, Design, and Simulation; IEEE Press, Wiley-Interscience: Piscataway, NJ, USA, 1992.

29. Buso, S.; Mattavelli, P. Digital Control in Power Electronics, 2nd ed.; Morgan \& Claypool: San Rafael, CA, USA, 2015.

30. Arrillaga, J.; Smith, B.C.; Watson, N.R.; Wood, A.R. Power System Harmonic Analysis; Wiley \& Sons Ltd.: Chichester, UK, 1997.

31. Black, H.S. Modulation Theory; Van Nostrand: Princeton, NJ, USA, 1953.

Publisher's Note: MDPI stays neutral with regard to jurisdictional claims in published maps and institutional affiliations.

(C) 2020 by the authors. Licensee MDPI, Basel, Switzerland. This article is an open access article distributed under the terms and conditions of the Creative Commons Attribution (CC BY) license (http:/ / creativecommons.org/licenses/by/4.0/). 